\title{
Efficient Analysis of Arbitrarily Shaped Inductive Obstacles in Rectangular Waveguides Using a Surface Integral-Equation Formulation
}

\author{
Fernando D. Quesada Pereira, Associate Member, IEEE, Vicente E. Boria Esbert, Senior Member, IEEE, \\ Juan Pascual García, Student Member, IEEE, Ana Vidal Pantaleoni, Member, IEEE, \\ Alejandro Alvarez Melcón, Member, IEEE, J. Luis Gomez Tornero, Member, IEEE, and \\ Benito Gimeno, Member, IEEE
}

\begin{abstract}
In this paper, we propose to use the surface integral-equation technique for the analysis of arbitrarily shaped $H$-plane obstacles in rectangular waveguides, which can contain both metallic and/or dielectric objects. The Green's functions are formulated using both spectral and spatial images series, whose convergence behavior has been improved through several acceleration techniques. Proceeding in this way, the convergence of the series is not attached to the employment of any particular basis or test function, thus consequently increasing the flexibility of the implemented technique. In order to test the accuracy and numerical efficiency of the proposed method, results for practical microwave circuits have been successfully compared with other numerical approaches.
\end{abstract}

Index Terms-Dielectric resonators, Green's functions, integral equations (IEs), waveguide components, waveguide discontinuities.

\section{INTRODUCTION}

$\mathbf{T}$ HE ANALYSIS and design of rectangular waveguide devices based on inductive obstacles is a subject extensively treated in the technical literature due to their wide use in space and ground telecommunications applications [1]-[4]. For the analysis of these problems, equivalent-circuit techniques were successfully derived in the past [5]. More recently, full-wave mode-matching methods [6]-[8] have been successfully applied to cope with these types of problems. However, for design purposes, more efforts should be devoted in order to improve the numerical efficiency of such analysis techniques, while preserving accuracy issues at the same time. To reach this aim, the integral-equation (IE) technique can be considered as a good alternative analysis method.

Manuscript received March 31, 2006; revised December 20, 2006. This work was supported by the Ministerio de Educación y Ciencia, Spanish Government under the coordinated Research Project TEC2004/04313-C02, and by Fundación Séneca 02972/PI/05.

F. D. Quesada Pereira, J. P. García, A. Alvarez Melcón, and J. L. Gomez Tornero are with the Communications and Information Technologies Department, Universidad Politcénica de Cartagena, Cartagena E-30202, Spain (e-mail: fernando.quesada@upct.es).

V. E. Boria Esbert and A. Vidal Pantaleoni are with the Departamento de Comunicaciones, Universidad Politécnica de Valencia, Valencia E-46022, Spain (e-mail: vboria@dcom.upv.es).

B. Gimeno is with the Departamento de Física Aplicada y Electromagnetismo, Universidad de Valencia, Valencia E-46100, Spain (e-mail: benito.gimeno@uv.es).

Digital Object Identifier 10.1109/TMTT.2007.893673
For instance, in [9], an IE method is used to derive equivalent circuits for a single metallic inductive square post. The analysis of an arbitrary number of metallic posts inside rectangular waveguides has also been the subject of other classical studies [10]-[12], where different IE approaches were successfully proposed. In these methods, the parallel-plate waveguide (PPW) Green's functions were used to reduce the numerical solution of the inductive problem. However, the PPW Green's functions consists of slowly convergent infinite series either in the spatial or in the spectral domain. To accelerate the convergence rate of such series, the Kummer's transformation was applied in [10] and [12], whereas particular choices of basis functions were successfully proposed in [11]. Nevertheless, the flexibility on the kind of geometries that could be treated in all such studies was widely reduced.

Later on, several extensions of previous IE methods for dealing with multiple dielectric obstacles were reported. For instance, in [13], a volume IE method solved by the method of moments (MoM) was successfully applied to the analysis of multiple dielectric posts. Bandpass and band-rejection filters based on two dielectric posts were fully designed in [14]. More recently, the IE technique was proposed for dealing with cavity filters loaded with dielectric and metallic posts [15], [16]. IE techniques have also been proposed for the study of multilayered media shielded planar printed circuits [17]. Although these techniques are efficient for planar metallizations, they become very complex when currents are directed along the stratified axis. Therefore, they are inappropriate for the study of inductive waveguide components. All these efforts suggest that further research on improving the flexibility and numerical efficiency of IE-based methods is needed for computer-aided design (CAD) purposes.

In this paper, the authors propose a very efficient surface IE technique for the accurate analysis of arbitrarily shaped inductive waveguide structures, which can be composed of any number of conducting and/or dielectric obstacles. A detailed study of the convergence behavior of both spatial and spectral domain series is presented, and new improvements regarding the fast evaluation of the PPW Green's functions and their spatial derivatives are proposed. This new approach allows the use of triangular functions in the MoM solution of the corresponding IEs, thus increasing the accuracy of the final results, as well as the complexity of the geometries that can be considered. Several waveguide devices of great practical interest, such as rod 


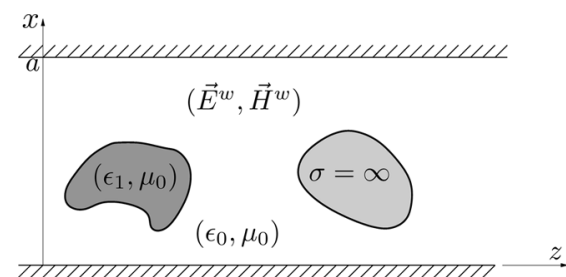

Fig. 1. Typical inductive geometry considered in this paper, which contains metallic and dielectric obstacles.

microwave filters and dielectric loaded filters including mechanization effects (i.e., the typical rounded corners effects), have been successfully considered for verification purposes.

\section{THEORY}

Conducting and dielectric inductive obstacles placed within a rectangular waveguide, as shown in Fig. 1, can be studied using a surface IE formulation [18]. Following this approach, two distinct, but coupled, problems are formulated. The first problem is called the external problem since it is formulated outside of the dielectric and metallic objects. The second problem is called the internal problem, since it is formulated inside of the dielectric objects. It is worth noticing that the internal problem does not need to be formulated inside the metallic objects since the fields are zero inside a perfect conductor region. For the treatment of the interior problem, the Green's functions are formulated as simple Hankel functions of second kind [19], while for the exterior problem, the PPW Green's functions will be used.

The proposed IE technique is based on the imposition of the boundary conditions for the electromagnetic fields. For the conducting bodies, a zero tangent component for the electric field is enforced. For the dielectric materials, we impose the continuity of the tangential components of the total electric and magnetic fields across the surfaces. This leads to the so-called Poggio-Miller-Chang-Harrington-Wu-Tsu (PMCHWT) formulation [20].

The system of IEs is then numerically solved following the well-known Galerkin-MoM technique. In this study, subsectional triangular basis functions [18] are used. The use of triangular functions is easy with this formulation since the Green's functions are directly obtained in the spatial domain. This strategy is useful for the analysis of complex shaped structures, and it is not possible in other spectral-domain approaches [11].

For numerical stability issues, the IEs are formulated using the mixed potential form. The mixing of the potentials leads to the need for the calculation of the spatial derivatives of the basic mixed potential components. As a consequence, the evaluation of the spatial derivatives of the Green's functions cannot be avoided in this formulation. In order to speed up the numerical evaluation of all Green's functions and derivatives, we will next study their convergence properties, and we will then derive a novel strategy for the efficient evaluation of the required series.

\section{A. PPW Green's Functions Convergence}

The PPW Green's functions in the structure shown in Fig. 2 can be formulated using the classical theory of images with respect to two parallel metallic ground planes [19]. In this case,

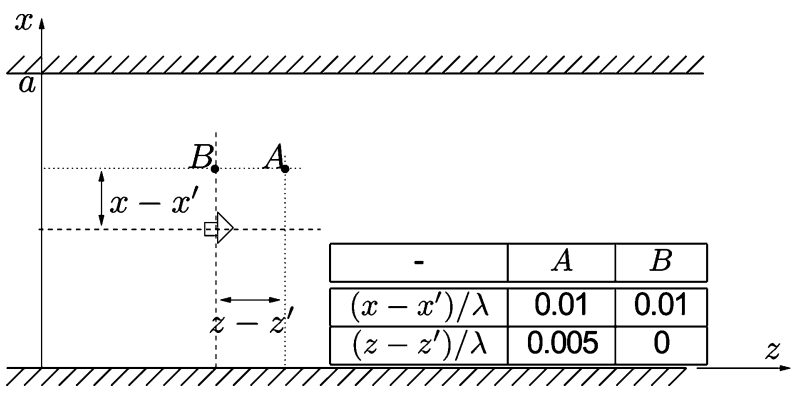

Fig. 2. Source and observer points locations in a PPW used in the convergence study $(a / \lambda=0.75)$.

TABLE I

VALUES OF THE PARAMETERS IN (1)-(4) FOR ALL RELEVANT GREEN'S FUNCTIONS COMPONENTS

\begin{tabular}{|c|c|c|c|c|}
\hline- & $s_{g}$ & $\xi$ & $f_{n}$ & $g_{n}$ \\
\hline \hline$G_{A}^{(e x t)}$ & -1 & $\mu_{0}$ & $\sin \left(k_{x} x\right)$ & $\sin \left(k_{x} x^{\prime}\right)$ \\
\hline$G_{W}^{(e x t)}$ & +1 & $1 / \epsilon_{0}$ & $\cos \left(k_{x} x\right)$ & $\cos \left(k_{x} x^{\prime}\right)$ \\
\hline
\end{tabular}

the scalar Green's function is expressed with the following spatial image series:

$$
\begin{aligned}
G_{\text {Spat }}^{(\text {ext })}\left(x, x^{\prime}, z-z^{\prime}\right)=\sum_{n=-\infty}^{+\infty}[ & G\left(x,+x^{\prime}+2 n a, z-z^{\prime}\right) \\
& \left.+s_{g} G\left(x,-x^{\prime}+2 n a, z-z^{\prime}\right)\right]
\end{aligned}
$$

where

$$
\begin{aligned}
G\left(x, x^{\prime}, z-z^{\prime}\right) & =\frac{\xi}{j 4} H_{0}^{(2)}\left(k_{0} \rho\right) \\
\rho & =\sqrt{\left(x-x^{\prime}\right)^{2}+\left(z-z^{\prime}\right)^{2}}
\end{aligned}
$$

$s_{g}$ is the sign function (see Table I), and $\xi$ is a constitutive parameter taking the values shown in Table I for the different Green's function components.

The spectral formulation of the PPW Green's functions is related to the spatial image series through the Poisson's summation formula [21]

$$
\sum_{n=-\infty}^{+\infty} f(2 n a)=\frac{\sqrt{2 \pi}}{2 a} \sum_{n=-\infty}^{+\infty} \tilde{f}(n \pi / a)
$$

where $(\tilde{f})$ denotes the Fourier transformation of $(f)$. Applying the above transformation, the spectral series takes the following general expression:

$$
\begin{aligned}
G_{\text {Spec }}^{(\text {ext })}\left(x, x^{\prime}, z-z^{\prime}\right) & \\
= & \frac{2}{a \pi} \sum_{n=0}^{\infty} \tilde{G}\left(k_{x}, z-z^{\prime}\right) f_{n}\left(k_{x} x\right) g_{n}\left(k_{x} x^{\prime}\right)
\end{aligned}
$$

where $f_{n}$ and $g_{n}$ are harmonic functions taking the form shown in Table I for the different Green's functions components, and the Fourier transformation of the Hankel function is

$$
\begin{aligned}
\tilde{G}\left(k_{x}, z-z^{\prime}\right) & =\xi \frac{e^{-j k_{z}\left|z-z^{\prime}\right|}}{j k_{z}} \\
k_{z} & =\sqrt{k_{0}^{2}-k_{x}^{2}} \\
k_{x} & =\frac{n \pi}{a} .
\end{aligned}
$$


TABLE II

VALUES OF THE PARAMETERS IN (5) AND (7) FOR THE Spatial Derivatives of THE GREEN's FunCtions

\begin{tabular}{|c|c|c|c|}
\hline- & $\xi$ & $f_{n}$ & $g_{n}$ \\
\hline \hline$\frac{\partial G_{A}^{(e x t)}}{\partial x}$ & $\mu_{0}$ & $\cos \left(k_{x} x\right)$ & $\sin \left(k_{x} x^{\prime}\right)$ \\
\hline$\frac{\partial G_{W}^{(e x t)}}{\partial x}$ & $1 / \epsilon_{0}$ & $-\sin \left(k_{x} x\right)$ & $\cos \left(k_{x} x^{\prime}\right)$ \\
\hline$\frac{\partial G_{A}^{(e x t)}}{\partial z}$ & $\mu_{0}$ & $\sin \left(k_{x} x\right)$ & $\sin \left(k_{x} x^{\prime}\right)$ \\
\hline
\end{tabular}

Due to the exponential behavior of this last expression, the convergence of the modal series is fast for large $\left|z-z^{\prime}\right|$ distances. On the contrary, the spatial images series in (1) exhibits much slower convergence behavior.

As it was introduced before, the spatial derivatives of the Green's functions are needed in this formulation. When the spatial images formulation is used, the corresponding derivatives only affect the Hankel term of the Green's function shown in (2a), which after simple calculations, give place to the following expressions:

$$
\begin{aligned}
& \frac{\partial G\left(x, x^{\prime}, z-z^{\prime}\right)}{\partial x}=-\frac{\xi}{j 4} k_{0}\left(x-x^{\prime}\right) \frac{H_{1}^{(2)}\left(k_{0} \rho\right)}{\rho} \\
& \frac{\partial G\left(x, x^{\prime}, z-z^{\prime}\right)}{\partial z}=-\frac{\xi}{j 4} k_{0}\left(z-z^{\prime}\right) \frac{H_{1}^{(2)}\left(k_{0} \rho\right)}{\rho}
\end{aligned}
$$

where now, the Hankel function of order 1 must be used inside the series representation of (1).

From the above expressions, we can readily notice the additional convergence factor due to the distance from the source point to the observation point $(\rho)$, which appears in the denominator. However, for the derivative along the $x$-axis, this additional $(\rho)$ term is compensated with the $\left(x-x^{\prime}\right)$ factor in the numerator. In fact, when the images distance increases, the global term $\left[\left(x-x^{\prime}\right) / \rho\right]$ tends to 1 . Therefore, convergence is only due to the first-order Hankel function. A different situation appears for the derivative along the $z$-axis. In this case, the factor $\left(z-z^{\prime}\right)$ remains constant when the distance of the images is increased. Consequently, the additional $(\rho)$ term results in a very important convergence rate improvement.

When similar derivatives are applied to the spectral representation of the series shown in (4), the following final expressions are obtained:

$$
\begin{aligned}
& \frac{\partial G^{(\mathrm{ext})}\left(x, x^{\prime}, z-z^{\prime}\right)}{\partial x} \\
& =+\frac{2}{a \pi} \sum_{n=0}^{\infty} \tilde{G}\left(k_{x}, z-z^{\prime}\right) k_{x} f_{n}\left(k_{x} x\right) g_{n}\left(k_{x} x^{\prime}\right) \\
& \frac{\partial G^{(\mathrm{ext})}\left(x, x^{\prime}, z-z^{\prime}\right)}{\partial z} \\
& \quad=-\frac{2}{a \pi} \sum_{n=0}^{\infty} \tilde{G}\left(k_{x}, z-z^{\prime}\right) j k_{z} f_{n}\left(k_{x} x\right) g_{n}\left(k_{x} x^{\prime}\right)
\end{aligned}
$$

where the spectral Green's function $(\tilde{G})$ is the same as in (5a), and the new harmonic functions change into the form shown in Table II for each corresponding component.

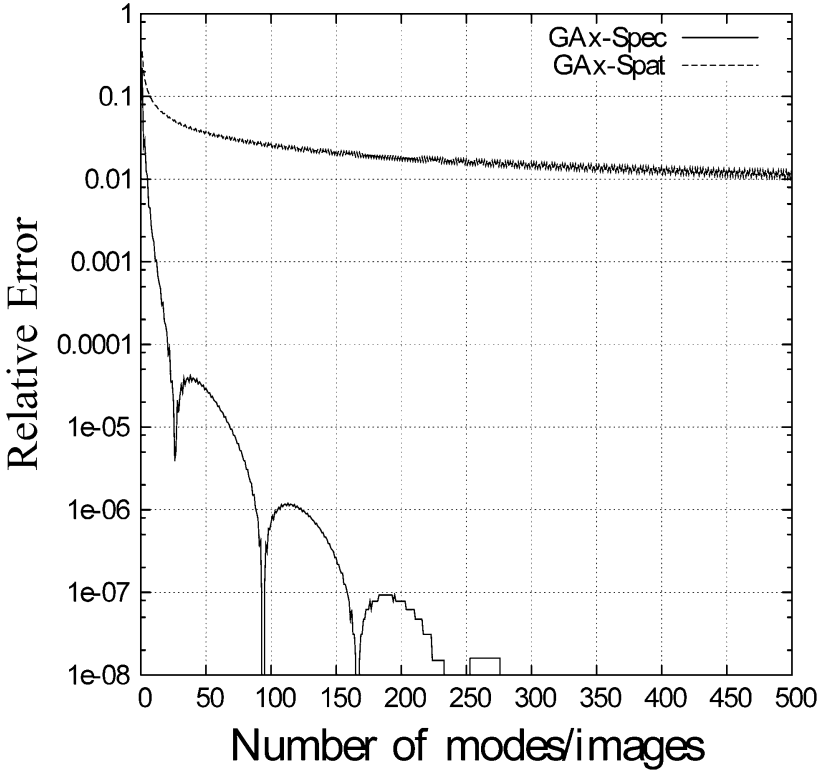

Fig. 3. Convergence of the spectral-domain series after application of the Kummer's transformation. Convergence of spatial-domain series are presented for comparison. Observer point at position $A$.

In this case, we can notice that convergence is degraded by the new spectral factors introduced by the derivatives. Therefore, a slower convergence rate is expected for the Green's functions derivatives using the spectral formulation.

In order to increase the convergence rate of the spectral series, the use of the Kummer transformation is proposed (see [10] and [22]). In the Appendix, we extend the Kummer formulation to all Green's functions and their spatial derivative components that appear following the surface IE derived in this paper. Now we perform a convergence behavior study after application of the Kummer's transformation to the spectral-domain formulation. In Figs. 3 and 4, we present the relative errors obtained for the basic Green's function components when the observer point is located at positions $A$ and $B$ (see Fig. 2). If we compare the behavior with the image series, we observe that convergence rate is strongly improved for observer point $A$. Now, even for the $B$ observer point (placed at the critical plane $z=z^{\prime}$ ), the spectral-domain series surpass largely the spatial images counterpart. By means of the employment of the Kummer's transformation, a good convergence behavior can be obtained at all points of the geometry (relative errors below $10^{-5}$ are always obtained with less than 100 modes).

To complete the study, we present in Figs. 5 and 6 similar convergence results, but now for the relevant spatial derivatives. With respect to the $z$-spatial derivative, we observe in Fig. 5 that the images series exhibits an impressive convergence behavior (only 15 images are needed to obtain a relative error of $10^{-5}$ ). Therefore, the spatial images series is preferred for the $z$-axis spatial derivative over the spectral representation. On the other hand, Fig. 6 shows that the Kummer technique is slightly better that the images series for the $x$-axis spatial derivative evaluated at the critical point $B\left(\left|z-z^{\prime}\right|=0\right)$. In this case, a relative error of $10^{-3}$ can be obtained with 100 modes using the Kummer transformation. If lower errors are needed for a 


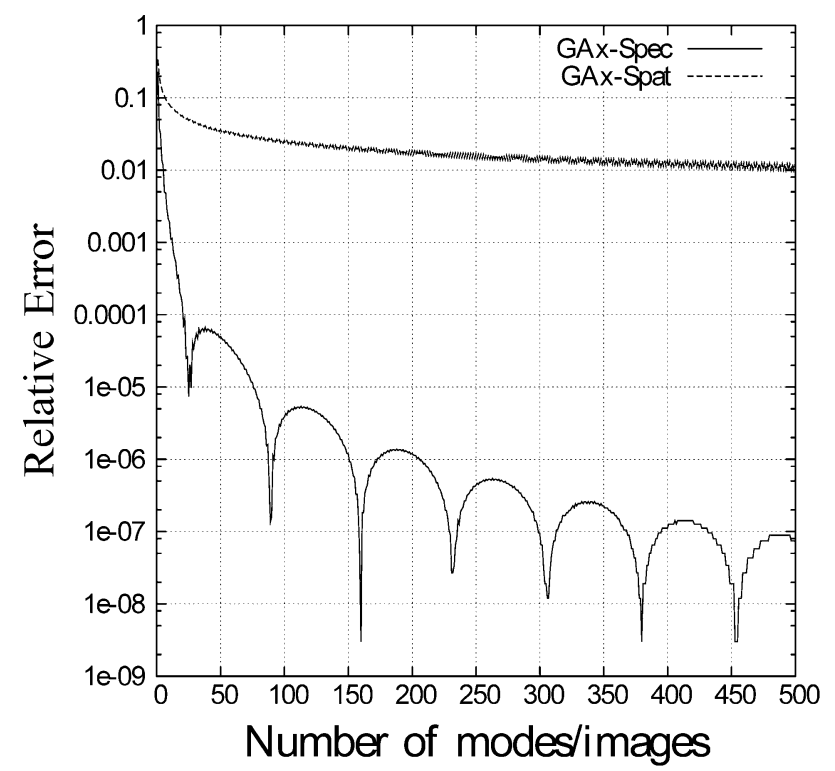

Fig. 4. Convergence of the spectral-domain series after application of the Kummer's transformation. Convergence of spatial-domain series are presented for comparison. Observer point at position $B$.

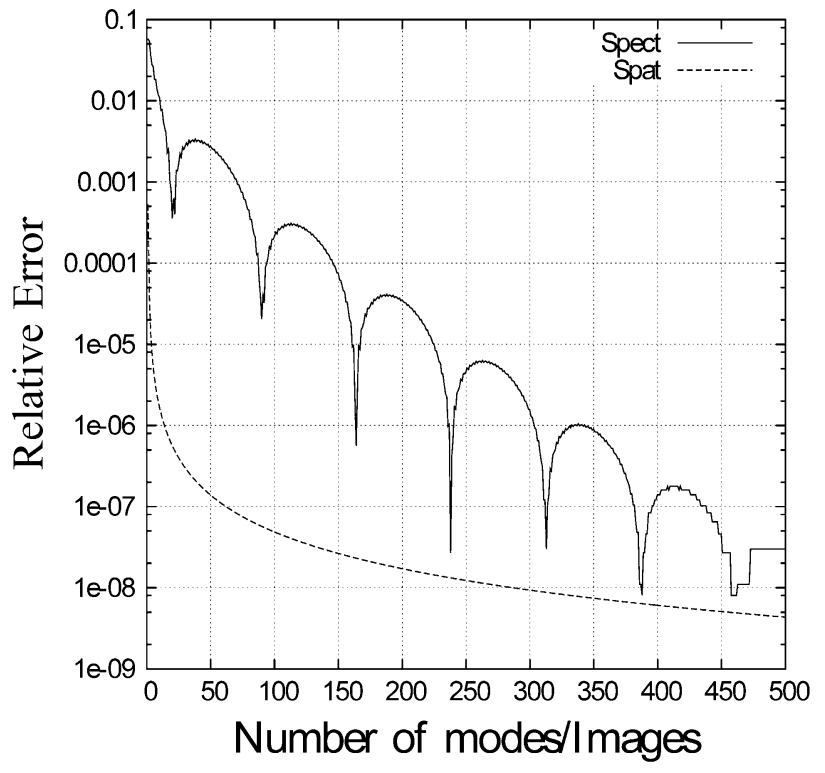

Fig. 5. Convergence of the spectral-domain spatial derivatives series after application of the Kummer transformation. Convergence of spatial-domain series for the derivatives are presented for comparison. Observer point at position $A$; spatial derivatives with respect to the $z$-axis.

particular application, other acceleration techniques can still be used, such as the Ewald method [23]. However, the use of the Ewald technique for all Green's function components and for all source-observer combinations is not recommended. Although the Ewald technique exhibits fast convergence behavior, it needs the evaluation of complementary error functions of complex arguments. The calculation of the complex complementary error function is slow so the overall computational cost is higher than with a clever combination of different techniques.

From the above convergence study, we can conclude that the PPW Green's functions and their spatial derivatives will be evaluated using the spectral representation of the series in combination with the Kummer transformation. This is the general rule,

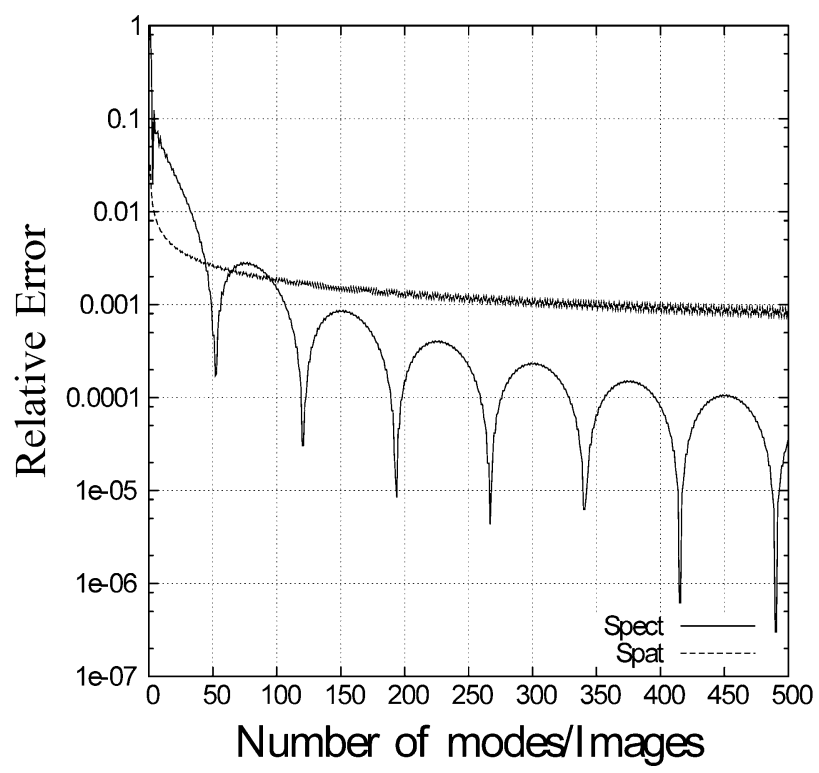

Fig. 6. Convergence of the spectral-domain spatial derivatives series after application of the Kummer's transformation. Convergence of spatial-domain series for the derivatives are presented for comparison. Observer point at position $B$; spatial derivatives with respect to the $x$-axis.

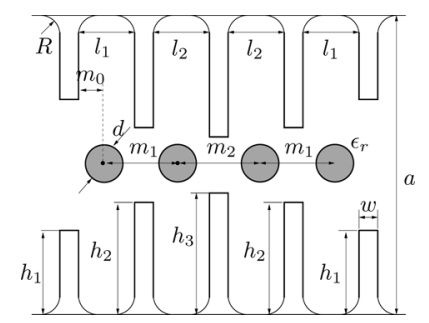

Fig. 7. Fourth-order dielectric loaded inductive filter. The dimensions are $a=$ $19.05 \mathrm{~mm}, w=2 \mathrm{~mm}, d=4.0 \mathrm{~mm}, \epsilon_{r}=2, h_{1}=4.725 \mathrm{~mm}, h_{2}=$ $6.405 \mathrm{~mm}, h_{3}=6.595 \mathrm{~mm}, l_{1}=12.03 \mathrm{~mm}, l_{2}=13.62 \mathrm{~mm}, m_{0}=$ $6.015 \mathrm{~mm}, m_{1}=14.815 \mathrm{~mm}$, and $m_{2}=15.600 \mathrm{~mm}, R=2.5 \mathrm{~mm}$.

except for the $z$ derivatives of the Green's functions at points satisfying the condition $\left|z-z^{\prime}\right| / \lambda<0.02$. We have seen in Fig. 5 that, in this region, the images series converges very fast and it is, therefore, preferred over the Kummer technique. Using this novel strategy, a very efficient formulation of the surface IE has been implemented.

\section{RESULTS}

To show the validity and flexibility of the IE technique just presented, we have investigated two inductive microwave waveguide filters of practical interest. For comparative purposes, all CPU times included here have been obtained on a PC with a 3-GHz CPU processor.

The first structure investigated is an inductively coupled rectangular waveguide filter with reduced dimensions, due to the use of dielectric posts inside the cavities (see Fig. 7). The typical rounded corners mechanization effects, attributed to low-cost milling manufacturing techniques, have also been considered (radius of curvature $R=2.5 \mathrm{~mm}$ ). The results are presented in Fig. 8, together with Ansoft's High Frequency Structure Simulator (HFSS) results, showing very good agreement with our new IE technique. For the simulation of this structure, we have 


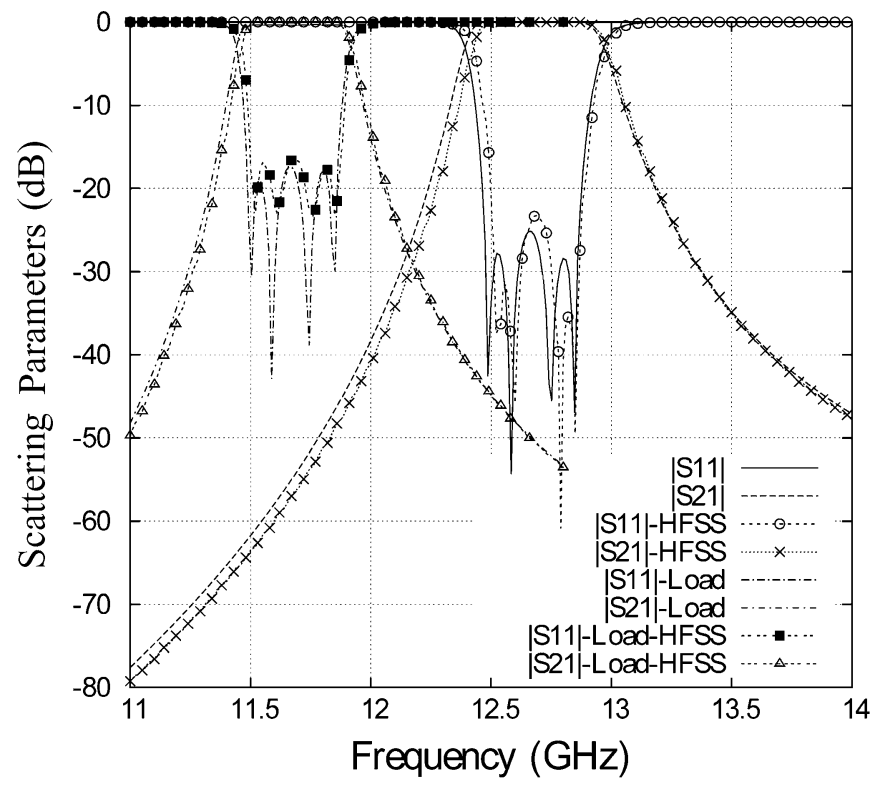

Fig. 8. Scattering parameters of the filter shown in Fig. 7 (load curves). Results for the filter without the posts are also included to show the loading effect of the posts.

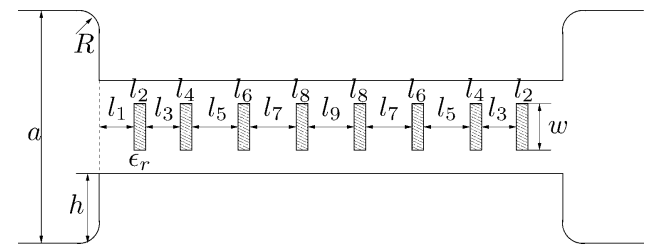

Fig. 9. Eighth-order evanescent rectangular waveguide filter composed of square dielectric posts. The dimensions are $a=28.5 \mathrm{~mm}, w=1.935 \mathrm{~mm}$, $h=8.825 \mathrm{~mm}, \epsilon_{r}=44, l_{1}=1.986 \mathrm{~mm}, l_{2}=0.899 \mathrm{~mm}, l_{3}=11.69 \mathrm{~mm}$, $l_{4}=0.951 \mathrm{~mm}, l_{5}=13.106 \mathrm{~mm}, l_{6}=0.95 \mathrm{~mm}, l_{7}=13.475 \mathrm{~mm}$, $l_{8}=0.95 \mathrm{~mm}$, and $l_{9}=13.56 \mathrm{~mm}, R=2 \mathrm{~mm}$.

used 178 basis functions, involving a computational effort of $0.98 \mathrm{~s} /$ frequency point. The results obtained with HFSS required a CPU time of $19.08 \mathrm{~s} /$ frequency point.

Finally, we have designed an evanescent rectangular waveguide filter composed of rectangular lossy dielectric posts (see Fig. 9). Inside the below cutoff waveguide of this structure, eight square dielectric resonators are coupled by proximity. Rounded corners are also considered during the mechanization of the below cutoff waveguide section, as shown in Fig. 9 (radius of curvature $R=2 \mathrm{~mm}$ ). In Fig. 10, we can observe the scattering parameters in the passband of the filter for several values of the dielectric loss tangent. As expected, the losses in the dielectric resonators essentially affect the insertion loss of the filter. In Table III, we show the minimum insertion loss achieved inside the passband for different values of the dielectric loss tangent.

It can be seen that the insertion loss can be kept below $0.5 \mathrm{~dB}$ if high-quality dielectric resonators are used with loss tangent values less than 0.0005 .

For validation purposes, we compare these results with the ones provided by the commercial software HFSS for the case of $\tan (\delta)=0.002$. A very good agreement is again obtained with the technique proposed in this paper. In particular, both numerical techniques predict exactly the same minimum insertion loss

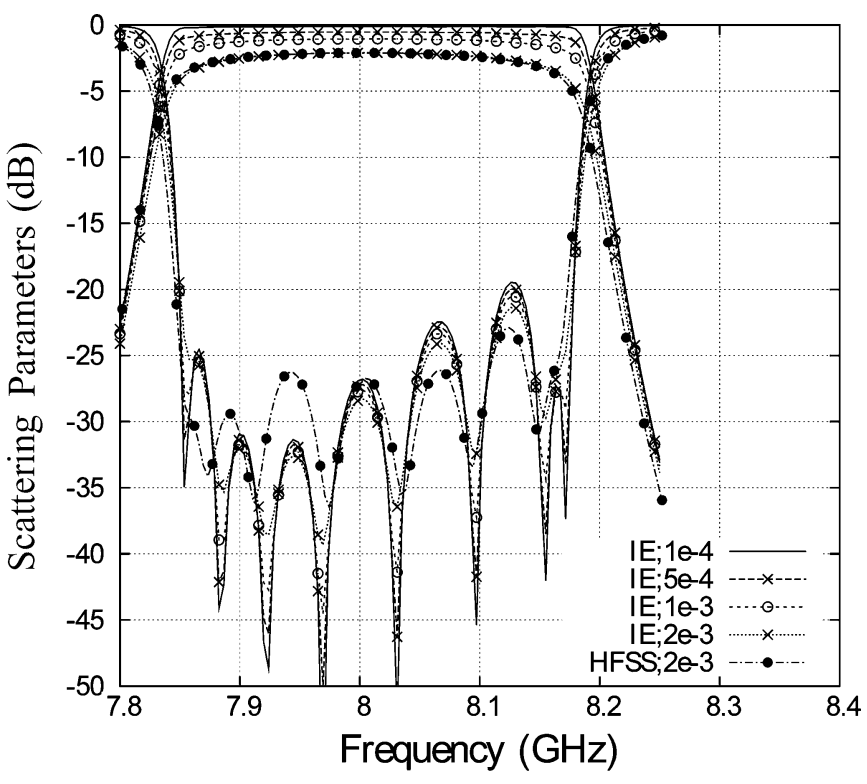

Fig. 10. In-band response of the filter shown in Fig. 9. Results provided by HFSS are included for comparison.

TABLE III

Minimum INSERTION LOSS WiTHIN THE PASSBAND FOR DifFERENT VALUES OF THE DIELECTRIC LOSS TANGENT

\begin{tabular}{|c|c|}
\hline $\tan (\delta)$ & Insertion Loss $(\mathrm{dB})$ \\
\hline \hline 0.0001 & 0.10 \\
\hline 0.0005 & 0.52 \\
\hline 0.001 & 1.05 \\
\hline 0.002 & 2.10 \\
\hline
\end{tabular}

within the passband (2.1 dB for the case of $\tan (\delta)=0.002)$. For the analysis of this evanescent waveguide filter, we have used 340 basis functions for segmenting the whole geometry. Our software tool has only taken $2.6 \mathrm{~s} /$ frequency point to complete the analysis, whereas the commercial software HFSS did require $39.6 \mathrm{~s} /$ frequency point to obtain the results with a similar degree of accuracy.

\section{CONCLUSION}

In this paper, a surface IE technique, based on PPW Green's functions, has been employed for the accurate and efficient analysis of arbitrarily shaped inductive obstacles placed within rectangular waveguides. To speed up the analysis tool, a deep study on the convergence properties of the Green's functions and their derivatives has been performed. Such study has led to a novel strategy, which combines the spatial series with the Kummer transformation applied to the spectral series. In this paper, several inductive filtering topologies of practical interest have been successfully studied using the new proposed technique.

\section{APPENDIX \\ KUMMER TRANSFORMATION FOR GREEN'S FUNCTIONS}

The Kummer transformation is based on the extraction of the asymptotic term of the series to be evaluated. Once extracted, 
the asymptotic term must be computed analytically. The extraction of the asymptotic term of the spectral series for the magnetic vector potential Green's function can be found in [22]. For the formulation presented in this study, we also need to accelerate the magnetic scalar potential Green's function shown in Table I. After some manipulations, the asymptotic term can be obtained in closed form as follows:

$$
\begin{aligned}
\sum_{n=1}^{\infty} \frac{j a}{n \pi} \cos \left(\frac{n \pi}{a} x\right) \cos \left(\frac{n \pi}{a} x^{\prime}\right) e^{-j(n \pi / a)\left|z-z^{\prime}\right|} & =-\frac{j a}{2 \pi} \operatorname{Re}\{\ln [(1-D)(1-C)]\} \\
C & =\exp \left\{j \frac{\pi}{a}\left[\left(x-x^{\prime}\right)+j\left|z-z^{\prime}\right|\right]\right\} \\
D & =\exp \left\{j \frac{\pi}{a}\left[\left(x+x^{\prime}\right)+j\left|z-z^{\prime}\right|\right]\right\} .
\end{aligned}
$$

In [22], the Kummer transformation is also applied to calculate some of the derivatives needed in the current formulation, as shown in Table II. In particular, in [22], we find the asymptotic terms for the $x$ and $z$ derivatives of the magnetic vector potential $\left(G_{A}^{(\mathrm{ext})}\right)$. Using the current formulation, we also need to evaluate in closed form the asymptotic term for the $x$ derivative of the magnetic scalar potential $\left(G_{W}^{(\text {ext })}\right)$. With the results presented in [22] this is a simple task, leading to

$$
\begin{aligned}
& \sum_{n=1}^{\infty} \sin \left(\frac{n \pi}{a} x\right) \cos \left(\frac{n \pi}{a} x^{\prime}\right) e^{-j(n \pi / a)\left|z-z^{\prime}\right|} \\
& \quad=\frac{1}{2} \operatorname{Im}\left\{\frac{D^{\prime}-C^{\prime}}{\left(1-C^{\prime}\right)\left(1-D^{\prime}\right)}\right\} \\
& C^{\prime}=\exp \left\{j \frac{\pi}{a}\left[\left(x^{\prime}-x\right)+j\left|z-z^{\prime}\right|\right]\right\} \\
& D^{\prime}=\exp \left\{j \frac{\pi}{a}\left[\left(x+x^{\prime}\right)+j\left|z-z^{\prime}\right|\right]\right\} .
\end{aligned}
$$

The asymptotic terms required in our formulation are now analytically evaluated using the expressions shown in (8) and (9), together with those given in [22].

\section{ACKNOWLEDGMENT}

The authors would like to thank Alcatel Alenia Space, Madrid, Spain, for providing specifications of the dielectric loaded evanescent waveguide filter.

\section{REFERENCES}

[1] Y.-C. Shih, "Design of waveguide $E$-plane filters with all metal inserts," IEEE Trans. Microw. Theory Tech., vol. MTT-32, no. 7, pp. 695-704, Jul. 1984

[2] G. Conciauro, P. Arcioni, M. Bressan, and L. Perregrini, "Wideband modeling of arbitrarily shaped $H$-plane waveguide components by the 'boundary integral-resonant mode expansion method'," IEEE Trans. Microw. Theory Tech., vol. 44, no. 7, pp. 1057-1066, Jul. 1996.

[3] M. Guglielmi, P. Jarry, E. Kerherve, O. Roquebrun, and D. Schmitt, "A new family of all-inductive dual-mode filters," IEEE Trans. Microw. Theory Tech., vol. 49, no. 10, pp. 1764-1769, Oct. 2001.

[4] V. E. Boria, M. Bozzi, D. Camilleri, A. Coves, H. Esteban, B. Gimeno, M. Guglielmi, and L. Polini, "Analysis and design of all-inductive filters with dielectric resonators," in 33rd Microw. Conf., Munich, Germany, Oct. 7-9, 2003, vol. 3, pp. 1247-1250.

[5] T. Toyama and E. Sawado, "Functionals in the variational method applied to equivalent impedance matrix of metallic posts unsymmetrically positioned in a rectangular waveguide," IEEE Trans. Microw. Theory Tech., vol. 40, no. 8, pp. 1655-1660, Aug. 1992.
[6] R. R. Mansour and R. H. Macphie, "An improved transmission matrix formulation of cascaded discontinuities and its application to $E$-plane circuits," IEEE Trans. Microw. Theory Tech., vol. MTT-34, no. 12, pp. 1490-1498, Dec. 1986.

[7] J. M. Reiter and F. Arndt, "Rigorous analysis of arbitrarily shaped $H$ - and $E$-plane discontinuities in rectangular waveguides by a fullwave boundary contour mode-matching method," IEEE Trans. Microw. Theory Tech., vol. 43, no. 4, pp. 796-801, Apr. 1995.

[8] H. Esteban, S. Cogollos, V. Boria, A. A. San Blas, and M. Ferrando, "A new hybrid mode-matching/numerical method for the analysis of arbitrarily shaped inductive obstacles and discontinuities in rectangular waveguides," IEEE Trans. Microw. Theory Tech., vol. 50, no. 4, pp. 1219-1224, Apr. 2002.

[9] T. Rozzi, F. Moglie, A. Morini, W. Gulloch, and M. Politi, "Accurate fullband equivalent circuits of inductive posts in rectangular waveguide," IEEE Trans. Microw. Theory Tech., vol. 40, no. 5, pp. 1000-1009, May 1992.

[10] Y. Leviatan, P. G. Li, A. T. Adams, and J. Perini, "Single post inductive obstacle in rectangular waveguide," IEEE Trans. Microw. Theory Tech., vol. MTT-31, no. 10, pp. 806-812, Oct. 1983.

[11] P. G. Li, A. T. Adams, Y. Leviatan, and J. Perini, "Multiple post inductive obstacles in rectangular waveguide," IEEE Trans. Microw. Theory Tech., vol. MTT-32, no. 4, pp. 365-373, Apr. 1984.

[12] H. Auda and R. F. Harrington, "Inductive posts and diaphragms of arbitrary shape and number in a rectangular waveguide," IEEE Trans. Microw. Theory Tech., vol. MTT-32, no. 6, pp. 606-613, Jun. 1984.

[13] C.-I. G. Hsu and H. A. Auda, "Multiple dielectric posts in a rectangular waveguide," IEEE Trans. Microw. Theory Tech., vol. MTT-34, no. 8, pp. 883-891, Aug. 1986.

[14] R. Gesche and N. Löchel, "Two cylindrical obstacles in a rectangular waveguide-resonances and filter applications," IEEE Trans. Microw. Theory Tech., vol. 37, no. 6, pp. 962-968, Jun. 1989.

[15] F. Alessandri, M. Chiodetti, A. Giugliarelli, D. Maiarelli, G. Martirano, D. Schmitt, L. Vanni, and F. Vitulli, "The electric-field integral-equation method for the analysis and design of a class of rectangular cavity filters loaded by dielectric and metallic cylindrical pucks," IEEE Trans. Microw. Theory Tech., vol. 52, no. 8, pp. 1790-1797, Aug. 2004.

[16] V. Catina, F. Arndt, and J. Brandt, "Hybrid surface integral-equation/ mode-matching method for the analysis of dielectric loaded waveguide filters of arbitrary shape," IEEE Trans. Microw. Theory Tech., vol. 53, no. 11, pp. 3562-3567, Nov. 2005.

[17] G. V. Eleftheriades, J. R. Mosig, and M. Guglielmi, "A fast integral equation technique for shielded planar circuits defined on nonuniform meshes," IEEE Trans. Microw. Theory Tech., vol. 44, no. 12, pp. 2293-2296, Dec. 1996.

[18] A. F. Peterson, S. L. Ray, and R. Mittra, Computational Methods for Electromagnetics. Piscataway, NJ: IEEE Press, 1998, p. 451.

[19] C. A. Balanis, Advanced Engineering Electromagnetics. New York: Wiley, 1989.

[20] A. J. Poggio and E. K. Miller, Integral Equation Solutions of ThreeDimensional Scattering Problems. Oxford, U.K.: Pergamon, 1973.

[21] R. E. Collin, Field Theory of Guided Waves. New York: IEEE Press, 1991.

[22] Y. Leviatan and G. S. Sheaffer, "Analysis of inductive dielectric posts in rectangular waveguide," IEEE Trans. Microw. Theory Tech., vol. MTT-35, no. 1, pp. 48-59, Jan. 1987.

[23] F. Capolino, D. R. Wilton, and W. A. Johnson, "Efficient computation of the 2-D Green's function for 1-D periodic structures using the Ewald method," IEEE Trans. Antennas Propag., vol. 53, no. 9, pp. 2977-2984, Sep. 2005.

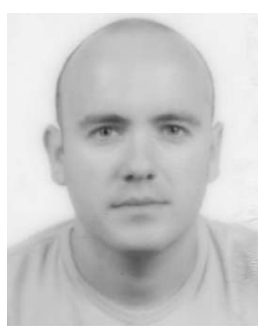

Fernando D. Quesada Pereira (S'05-A'07) was born in Murcia, Spain, in 1974. He received the Telecommunications Engineer degree from the Universidad Politécnica de Valencia (UPV), Valencia, Spain, in 2000, and is currently working toward the Ph.D. degree at the Universidad Politécnica de Cartagena (UPCT), Cartagena, Spain.

In 1999, he joined the Radiocommunications Department, UPV, as a Research Assistant, where he was involved in the development of numerical methods for the analysis of anechoic chambers and tag antennas. In 2001, he joined the Communications and Information Technologies Department, UPCT, initially as a Research Assistant, and then as an Assistant Professor. In 2005, he spent six months as a Visiting Scientist with the University of Pavia, Pavia, Italy. His current scientific interests include IE numerical methods for the analysis of antennas and microwave devices. 


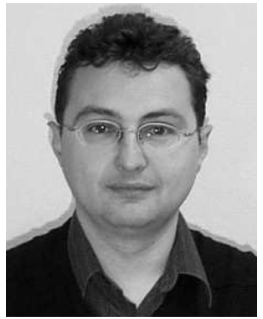

Vicente E. Boria Esbert (S'91-A'99-SM'02) was born in Valencia, Spain, on May 18, 1970. He received the Ingeniero de Telecomunicación degree (with first-class honors) and Doctor Ingeniero de Telecomunicación degree from the Universidad Politécnica de Valencia, Valencia, Spain, in 1993 and 1997, respectively.

In 1993, he joined the Departamento de Comunicaciones, Universidad Politécnica de Valencia, where since 2003 he has been a Full Professor. In 1995 and 1996, he was held a Spanish Trainee position with the European Space Research and Technology Centre (ESTEC)-European Space Agency (ESA), Noordwijk, The Netherlands, where he was involved in the area of electromagnetic (EM) analysis and design of passive waveguide devices. $\mathrm{He}$ has authored or coauthored five chapters in technical textbooks, 40 papers in refereed international technical journals, and over 100 papers in international conference proceedings. His current research interests include numerical methods for the analysis of waveguide and scattering structures, automated design of waveguide components, radiating systems, measurement techniques, and power effects (multipactor and corona) in waveguide systems.

Dr. Boria Esbert is a member of the IEEE Microwave Theory and Techniques Society (IEEE MTT-S) and the IEEE Antennas and Propagation Society (IEEE AP-S) since 1992. He serves on the Editorial Boards of the IEEE TRANSACTIONS ON MicROWAVE THEORY AND TECHNIQUES and IEEE MiCROWAVE AND WIRELESS COMPONENTS LETTERS. He is also a member of the Technical Committees of the IEEE MTT-S International Microwave Symposium (IMS) and of the European Microwave Conference. He was the recipient of the 2001 Social Council of Universidad Politécnica de Valencia First Research Prize for his outstanding activity during 1995-2000.

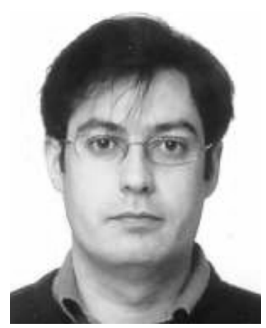

Juan Pascual García (S'05) was born in Castellón, Spain, in 1975. He received the Telecommunications engineer degree from the Universidad Politécnica de Valencia (UPV), Valencia, Spain, in 2001.

In 2003, he joined the Communications and Information Technologies Department, Universidad Politcénica de Cartagena (UPCT), Cartagena, Spain, where he is currently working toward the Ph.D. degree.

His research interests include neural networks, genetic algorithms, and their applications in the analysis and development of a CAD tool for microwave circuits and antennas.

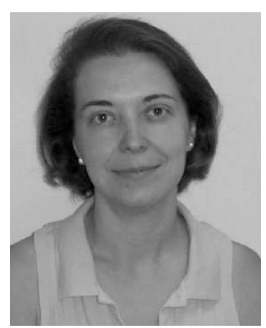

Ana Vidal Pantaleoni (M’01) was born in Valencia, Spain, in 1970. She received the Ingeniero de Telecomunicación degree from the Universidad Politécnica de Valencia, Valencia, Spain, in 1993.

In 1993, she spent one year with the University of Strathclyde, Glasgow, U.K., under the Erasmus international exchange program. In 1993, she was involved in broadband communications development in the main research center of Telecom Portugal. She then became a Research Assistant with the Universidad Politécnica de Valencia. In 1995 and 1996, she held a Spanish Trainee position with the European Space research and Technology Centre (ESTEC)-European Space Agency (ESA), Noordwijk, The Netherlands, where she was involved in the study and implementation of software for synthetic aperture radar (SAR) image processing. In 1996, she returned to the Universidad Politécnica de Valencia, where she held several lecturing positions, and became an Associate Professor in 2001. Her current interests are SAR data processing, SAR speckle noise reduction, and numerical methods for microwave structures analysis including the wavelet transform.

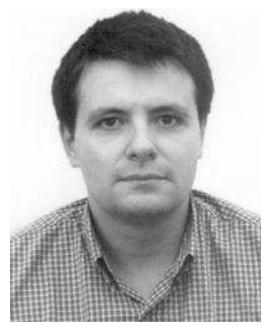

Alejandro Alvarez Melcón (M’99) was born in Madrid, Spain, in 1965. He received the Telecommunications Engineer degree from the Polytechnic University of Madrid (UPM), Madrid, Spain, in 1991, and the Ph.D. degree in electrical engineering from the Swiss Federal Institute of Technology, Lausanne, Switzerland, in 1998.

In 1988, he joined the Signal, Systems and Radiocommunications Department, UPM, as a Research Student, where he was involved in the design, testing, and measurement of broadband spiral antennas for electromagnetic measurements support (EMS) equipment. From 1991 to 1993, he was with the Radio Frequency Systems Division, European Space Agency (ESA/ESTEC), Noordwijk, The Netherlands, where he was involved in the development of analytical and numerical tools for the study of waveguide discontinuities, planar transmission lines, and microwave filters. From 1993 to 1995, he was with the Space Division, Industry Alcatel Espacio, Madrid, Spain, where he was involved with the ESA and collaborated in several ESA/ESTEC contracts. From 1995 to 1999, he was with the Swiss Federal Institute of Technology, École Polytechnique Fédérale de Lausanne, Lausanne, Switzerland, where he was involved in the field of microstrip antennas and printed circuits for space applications. In 2000, he joined the Universidad Politcénica de Cartagena (UPCT), Cartagena, Spain, where he currently develops his teaching and research activities.

Dr. Alvarez Melcón was the recipient of the Journée Internationales de Nice sur les Antennes (JINA) Best Paper Award for the best contribution to the JINA'98 International Symposium on Antennas, and the Colegio Oficial de Ingenieros de Telecomunicación (COIT/AEIT) Award for the best doctoral thesis in basic information and communication technologies.

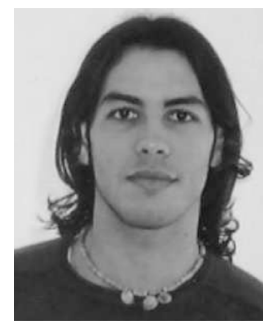

José Luis Gómez Tornero (M'06) was born in Murcia, Spain, in 1977. He received the Telecommunications Engineer degree from the Universidad Politécnica de Valencia (UPV), Valencia, Spain, in 2001, and the Ph.D. degree (laurea cum laude) in telecommunication engineering from the Universidad Politcénica de Cartagena (UPCT), Cartagena, Spain, in 2005.

In 1999, he joined the Radiocommunications Department, UPV, as a Research Student, where he was involved in the development of analytical and numerical tools for the study and automated design of microwave filters in waveguide technology for space applications. In 2000, he joined the Radio Frequency Division, Industry Alcatel Espacio, Madrid, Spain, where he was involved with the development of microwave active circuits for telemetry, tracking, and control (TTC) transponders implicated in many different spatial missions for the European Space Agency (ESA), National Aeronautics Space Administration (NASA), and other space agencies. In 2001, he joined the UPCT, as an Assistant Professor, where he currently develops his teaching activities. Since October 2005, he has been Vice Dean for students and lecture affairs with the Telecommunication Engineering Faculty, UPCT. His scientific research is focused on the analysis and design of leaky-wave antennas for millimeter-waveband applications and the development of numerical methods for the analysis of novel passive radiating structures in planar and waveguide technologies. His scientific interests also include the study of active devices for microwave and millimeter wavebands such as oscillators and active antennas.

Dr. Gómez Tornero was the recipient of the 2004 Second National Award presented by the EPSON-Ibérica Foundation for the best doctoral project in the field of technology of information and communications (TIC). He was also the recipient of the 2006 Vodafone Foundation Colegio Oficial de Ingenieros de Telecomunicación (COIT/AEIT) Award presented to the best Spanish doctoral thesis in the area of advanced mobile communications technologies.

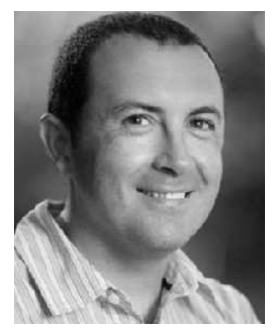

Benito Gimeno (M'01) was born in Valencia, Spain, on January 29, 1964. He received the Licenciado degree in physics and Ph.D. degree from the Universidad de Valencia, Valencia, Spain, in 1987 and 1992, respectively.

From 1987 to 1990, he was a Fellow with the Universidad de Valencia. Since 1990, he has been an Assistant Professor with the Departamento de Física Aplicada y Electromagnetismo, Universidad de Valencia, where in 1997 he became an Associate Professor. During 1994 and 1995, he was with the European Space Research and Technology Centre (ESTEC), European Space Agency (ESA), as a Research Fellow. In 2003, he was with the Università degli Studi di Pavia, Pavia, Italy, as a Visiting Scientist for a three-month period. His current research interests include computer-aided techniques for analysis of passive components for space applications, waveguides, and cavities including dielectric objects, electromagnetic-bandgap structures, frequency-selective surfaces, and nonlinear phenomena appearing in power microwave subsystems (multipactor and corona effects).

Dr. Gimeno was the recipient of a 2003 Spanish Government Fellowship. 